Oxford Preprint: OUTP 95/07P

9th March 1995

\title{
The Critical Exponents of Crystalline Random Surfaces
}

\author{
UKQCD Collaboration \\ J.F. Wheater \\ Theoretical Physics, 1 Keble Road, University of Oxford, Oxford OX1 3NP, UK
}

\begin{abstract}
We report on a high statistics numerical study of the crystalline random surface model with extrinsic curvature on lattices of up to $64^{2}$ points. The critical exponents at the crumpling transition are determined by a number of methods all of which are shown to agree within estimated errors. The correlation length exponent is found to be $\nu=0.71(5)$ from the tangenttangent correlation function whereas we find $\nu=0.73(6)$ by assuming finite size scaling of the specific heat peak and hyperscaling. These results imply a specific heat exponent $\alpha=0.58(10)$; this is a good fit to the specific heat on a $64^{2}$ lattice with a $\chi^{2}$ per degree of freedom of 1.7 although the best direct fit to the specific heat data yields a much lower value of $\alpha$. Our measurements of the normal-normal correlation functions suggest that the model in the crumpled phase is described by an effective field theory which deviates from a free field theory only by super-renormalizable interactions.
\end{abstract}

\footnotetext{
${ }^{1}$ e-mail: jfw@thphys.ox.ac.uk
} 


\section{Introduction}

The crystalline random surface model has attracted considerable interest over the past few years. Although such models have obvious applications in condensed matter physics [1] their role in the physics of two-dimensional quantum gravity is perhaps the most intriguing. For some time the nature of two-dimensional quantum gravity coupled to conformal matter with central charge $c \leq 1$ has been reasonably well understood [2, 3]; however this understanding fails when $c>1$. Roughly speaking when a small number of matter species are present the physics is a small perturbation from that when there is no matter; when the number of matter species exceeds a certain value the self coupling of gravity through intermediary matter fields becomes strong and the method of calculation fails. (Whether the physics changes abruptly is not yet properly understood.)

It is quite remarkable that there is an (apparently) exact lattice formulation of two-dimensional quantum gravity problems. In this formulation the functional integral over the metric is replaced by summing over all triangulations of the lattice [4, 5, 6] and matter fields are provided by placing a spin system on the lattice. At its critical point the model is the same as conformal matter (with $c$ equal to that of the spin system at its fixed lattice critical point) interacting with quantum gravity; this is known by direct calculation in the case of the Ising model for example [7]. In this approach the simplest way to obtain integer $c=D$ is to put $D$ free (that is, not self-interacting - they do of course interact through their coupling to the dynamical triangulation), massless bosonic fields on the lattice. The model is then mathematically equivalent to one of a random surface, which is allowed to self-intersect, embedded in $D$ dimensions. Unfortunately, it turns out that such models do not lead to a smooth continuum limit because there are no correlations between normals of the surface and the continuum string tension is always infinite [8]. To overcome this problem it was proposed to add an extrinsic curvature term to the action [9]; this introduces a correlation between normals and so might lead to a smoother surface with a finite string tension in the continuum limit. The resulting model on a fixed triangulation, usually called the crystalline surface model, has been shown to have a second order phase transition, as have similar models [10, 11, 12, 13, 14]. Of course, since this is no longer a free field theory, it is clear neither that the effective field theory governing the critical point is a conformal field theory nor, if it is, whether it actually has $c>1$. If this critical point is conformal with $c>1$ then the corresponding dynamically triangulated model might lead to a consistent theory of quantum gravity interacting with $c>1$ conformal matter.

There are several recent papers which review the status of random surface models per se [14, 15] and various approaches to two-dimensional quantum gravity [16, 17]. The purpose of this paper is to report on a high statistics numerical study of one model in $D=3$ dimensions in the region of its critical point; preliminary results which did not use the full data set analyzed here were presented in [13. This paper is organized as follows. In sections 2 and 3 we describe the model and our simulation methods, sections 4, 5 and 6 deal with the results and in section 7 we discuss our conclusions. 


\section{The model}

The crystalline surface model approximates a continuum surface by a (regular) triangulation with $N$ vertices labelled $i=1, \ldots N$; each vertex has a location on the lattice denoted by its two-component intrinsic coordinates $\vec{\xi}(i)$ and a location in the $D$-dimensional euclidean embedding space $\mathbf{X}(i)$. We will sometimes refer to the components of $\mathbf{X}$ as $X_{\mu}$ where $\mu=1, \ldots D$. In numerical work it is much easier to use closed surfaces and our choice of lattice boundary conditions (periodic in $y$ and helical in $x$ [12]) make the surface a torus.

There are several ways of assigning an action to this system [10, 11, 18] and for some time it appeared that they did not all have the same critical properties. However, recent work [14 has demonstrated that the analysis of simulations is complicated by very long autocorrelation times and that in fact all the models have similar critical behaviour. In this paper we work with the model whose action is given by

$$
S=\beta^{2} S_{G}+\kappa S_{e c}
$$

where $\beta$ and $\kappa$ are coupling constants. $S_{G}$ is gaussian and given by

$$
S_{G}=\frac{1}{2} \sum_{<i j>}(\mathbf{X}(i)-\mathbf{X}(j))^{2}
$$

where $\langle i j\rangle$ denotes the link joining neighbouring points labelled by $i$ and $j$. $S_{e c}$ denotes the extrinsic curvature action and is given by

$$
S_{e c}=-\sum_{<i j>} \hat{\mathbf{n}}_{\triangle} \cdot \hat{\mathbf{n}}_{\triangle^{\prime}}
$$

where $\triangle, \triangle^{\prime}$ are the triangles sharing the link $\langle i j\rangle, \mathbf{n}_{\triangle}$ is the normal vector of $\triangle$, and $\hat{\mathbf{n}}_{\triangle}$ the correspond unit vector [10]. The partition function is given by

$$
Z=\prod_{i=1}^{N} \int d^{D} \mathbf{X}(i) \delta^{D}\left(\sum_{i=1}^{N} \mathbf{X}(i)\right) e^{-S}
$$

where the delta function has been included to remove the translational zero mode. Expectation values are given by

$$
\langle\mathcal{O}\rangle=\frac{1}{Z} \prod_{i=1}^{N} \int d^{D} \mathbf{X}(i) \delta^{D}\left(\sum_{i=1}^{N} \mathbf{X}(i)\right) \mathcal{O}(\{\mathbf{X}\}) e^{-S}
$$

By exploiting the invariance of $S_{e c}$ under the global rescaling $\mathbf{X} \rightarrow \lambda \mathbf{X}$ it is easy to derive the results

$$
\begin{aligned}
\left\langle S_{G}\right\rangle & =\frac{(N-1) D}{2 \beta^{2}} \\
\left\langle S_{G}^{2}\right\rangle-\left\langle S_{G}\right\rangle^{2} \equiv\left\langle S_{G}^{2}\right\rangle_{c} & =\frac{(N-1) D}{2 \beta^{4}}
\end{aligned}
$$


and so on; note that $\beta$ can be scaled away entirely but we will retain it for later convenience.

At $\kappa=0$ this model is exactly soluble and describes a surface whose mean square radius

$$
\left\langle\mathbf{X}^{2}\right\rangle=\frac{1}{N} \sum_{i=1}^{N} \mathbf{X}(i) \cdot \mathbf{X}(i)
$$

behaves as

$$
\left\langle\mathbf{X}^{2}\right\rangle \sim \log N
$$

Defining a "Hausdorff" dimension $d_{H}$ through

$$
\left\langle\mathbf{X}^{2}\right\rangle \sim N^{\frac{2}{d_{H}}}
$$

the behaviour at $\kappa=0$ corresponds to $d_{H}=\infty$ and the surface is so highly crumpled that it is space filling in arbitrary dimension $D$; this behaviour persists at small $\kappa$ and this phase is usually called the "crumpled phase". The extrinsic curvature term, $S_{e c}$, acts to suppress sharp bends in the surface and so as $\kappa$ increases we expect the surface to become smoother. It is now well-established [10, 11, 12, 13] that there is a second order phase transition at some critical value, $\kappa_{c}$, of the coupling. There are reasons for believing that at $\kappa=\kappa_{c}$ the value of $d_{H}$ drops to 4 or less [11, 12]; as $\kappa \rightarrow \infty$ the surface becomes smoother and we expect $d_{H} \rightarrow 2$. In fact the numerical evidence [12] indicates that $d_{H}$ goes straight to 2 at the critical point.

Although considerable effort has gone into determining the nature of the critical point and measuring the critical exponents there are still some apparent discrepancies. The purpose of this work was to gather very high statistics on lattices of moderate size in order to see if the discrepancies could be resolved. As a byproduct, we have also been able to measure some quantities for the first time.

\section{The simulation}

There are some advantages to simulating these models using the Langevin method with Fourier acceleration. This is largely because the action depends only upon derivatives of the $\mathbf{X}_{\mathrm{s}}$ and not on the fields themselves; it has been observed [12] that a Fourier accelerated simulation gives much shorter autocorrelation times. Although this is partly offset by the extra computer time needed for the Fourier transforms there is still a substantial gain. However the method is not perfect and critical slowing down is still observed near the phase transition. The method of Fourier acceleration used in this work is identical to that described in [12] but another aspect of the algorithm has been changed.

In a Langevin simulation, new configurations are generated by discretizing the Langevin time $\tau$ in steps of $\delta \tau$; at an update the change in $\mathbf{X}$ is given by

$$
\delta X_{\mu}(i)=-\delta \tau \frac{\partial S}{\partial X_{\mu}(i)}+\eta(i, \tau) \sqrt{\delta \tau}
$$


where $\eta$ is a gaussian distributed random number satisfying $\left\langle\eta(i, \tau) \eta\left(j, \tau^{\prime}\right)\right\rangle=2 \delta_{i j} \delta_{\tau \tau^{\prime}}$. The derivative of $S_{e c}$ depends upon

$$
\frac{\partial \hat{\mathbf{n}}_{\triangle}}{\partial X_{\mu}(i)}=\frac{1}{\left|\mathbf{n}_{\triangle}\right|}\left(\frac{\partial \mathbf{n}_{\triangle}}{\partial X_{\mu}(i)}-\hat{\mathbf{n}}_{\triangle}\left(\hat{\mathbf{n}}_{\triangle} \cdot \frac{\partial \mathbf{n}_{\triangle}}{\partial X_{\mu}(i)}\right)\right)
$$

which is non-zero if $i$ is one of the vertices of $\Delta$ and zero otherwise. If $\Delta$ has a very small area $\left|\mathbf{n}_{\Delta}\right|$ then it is possible that a very large update for $X_{\mu}(i)$ will be generated; there is no reason why the expression in brackets in (12) should be particularly small when $\left|\mathbf{n}_{\Delta}\right|$ is small. Thus configurations of the surface which have one or more triangles of very small area are prone to generate large spikes at the next update. This problem exists for any finite $\delta \tau$ and any non-zero $\kappa$ and is an artefact of discretizing the Langevin equation. To deal with the difficulty it is necessary to modify the simulation procedure so as to suppress the contribution of triangles of very small area. This may be done by introducing a small area cut-off; the resulting procedure is rather like a hybrid algorithm. However this is computationally very inefficient to implement and we have developed a different method.

We work with the modified extrinsic curvature action

$$
\bar{S}_{e c}=-\sum_{<i j>} \hat{\mathbf{n}}_{\triangle} \cdot \hat{\mathbf{n}}_{\triangle^{\prime}} \exp \left(-\sigma \delta \tau\left(\frac{1}{\left|\mathbf{n}_{\triangle}\right|}+\frac{1}{\left|\mathbf{n}_{\triangle}^{\prime}\right|}\right)\right)
$$

where $\sigma$ is some constant so that the total action for the simulation is given by

$$
S_{s y m}=\beta^{2} S_{G}+\kappa \bar{S}_{e c} .
$$

When $\delta \tau \rightarrow 0$ this reduces (at least naively) to the original action. On the other hand at finite $\delta \tau$ the contribution of any triangle of area much less than $\sigma \delta \tau$ to the Langevin update is heavily suppressed. The exponential suppression wins over any power law from (12) and provides a cut-off which is almost a theta function but has the virtue of being differentiable; thus $\bar{S}_{e c}$ can be simulated by a completely standard Langevin method. (Note that this procedure does not suppress the creation of small triangles, it merely prevents them generating spikes.) Working with $\bar{S}_{e c}$ is also very efficient computationally; compared to the original action $S_{e c}$ very little extra calculation is needed. Simple analysis shows that $\sigma$ is dimensionless; in principal we could choose it to take any value but its presence can be exploited further. A Langevin simulation with finite $\delta \tau$ always renormalizes the couplings of the bare action and in general introduces other operators as well [20]. This has the consequence that the $\mathbf{X}$ configurations that we generate do not actually yield the expectation value (6) correctly because $\beta$ is renormalized; this trivial renormalization can be computed by comparing the measured and theoretical values of $\left\langle S_{G}\right\rangle$. However, even after taking account of the renormalization of $\beta$, higher moments such as (1) do not come out as predicted because other operators are induced by the discretization. We use the freedom to tune $\sigma$ to arrange that the measured ratio

$$
\rho=\frac{(N-1) D}{2}\left\langle S_{G}^{2}\right\rangle_{c} /\left\langle S_{G}\right\rangle^{2}
$$


is close to the predicted value of 1 ; that is to say we can remove at least some of the effects induced by the discretization of the Langevin time. We found that it is easy to tune $\sigma$ so that the residual discrepancy in $\rho$ is of order $1 \%$. The value of $\sigma$ needed is approximately independent of $\delta \tau$, as it should be since $[\sigma]=0$, at least over the range $\delta \tau=0.001$ to 0.004 which brackets the value of $\delta \tau=0.002$ used in our simulations as is shown in fig.1. That the procedure is consistent is shown in fig. 2 ; the value of $\sigma$ deduced by tuning on a $16^{2}$ lattice works just as well on a $64^{2}$ lattice. Higher moments of $S_{G}$ are still affected by discretization errors; for example the connected third moment comes out about 50\% too large (although it should be realized that it is very difficult to measure this double-subtracted quantity accurately).

Despite the improvements due to the Fourier acceleration it is still necessary to acquire very high statistics for reliable measurements. For example, in the case of the specific heat (defined in section 5) the total data set consisting of $T_{0}$ sweeps is split into $K$ bins; we then compute the specific heats, $C_{0}$ from the total data set, and $C_{k}(k=1, \ldots K)$ from each bin separately. We define $K_{b i n}$ to be the largest value of $K$ for which

$$
\left|1-\frac{1}{C_{0} K} \sum_{k=1}^{K} C_{k}\right|<0.01
$$

and then

$$
T_{b i n}=T_{0} K_{b i n}^{-1} .
$$

Subsequent analysis is then done using bins of size $T_{b i n}$; in this way we ensure that the statistical errors in the measured specific heat are larger than the thermalization errors. The values of $T_{b i n}$ found, measured in numbers of second order Runge-Kutta iterations of our algorithm, are shown in fig.3; as can be seen $T_{\text {bin }}$ appears to scale

roughly like $\sqrt{N}$ which is consistent with the behaviour found in [12]. We have run our simulations for at least $10 T_{\text {bin }}$ iterations except in the case of crucial $\kappa$ values where we have run for much longer; in every case the first $2 T_{\text {bin }}$ iterations are discarded. Thus we have accumulated about $10^{8}$ iterations of the algorithm in total.

\section{Tangent-Tangent Correlation Functions}

Much of the information about the long distance properties of the system is contained in the tangent-tangent correlation functions. Let

$$
\begin{aligned}
& \mathbf{t}_{1}(\vec{\xi}) \equiv \mathbf{t}_{1}\left(\xi_{1}, \xi_{2}\right)=\mathbf{X}\left(\xi_{1}+1, \xi_{2}\right)-\mathbf{X}\left(\xi_{1}, \xi_{2}\right) \\
& \mathbf{t}_{2}(\vec{\xi}) \equiv \mathbf{t}_{2}\left(\xi_{1}, \xi_{2}\right)=\mathbf{X}\left(\xi_{1}+1, \xi_{2}+1\right)-\mathbf{X}\left(\xi_{1}, \xi_{2}\right)
\end{aligned}
$$

then the correlation functions $G_{i j}$ are defined by

$$
G_{i j}(\xi)=\frac{1}{N} \sum_{\xi_{2}}\left\langle\mathbf{t}_{j}\left(\xi, \xi_{2}\right) \cdot \mathbf{t}_{i}\left(0, \xi_{2}\right)\right\rangle
$$


On a periodic lattice it follows that [12]

$$
\sum_{\xi} G_{11}(\xi)=\frac{1}{N} \sum_{\xi_{2}}\left\langle\left(\sum_{\xi} \mathbf{t}_{1}\left(\xi, \xi_{2}\right)\right) \cdot \mathbf{t}_{1}\left(0, \xi_{2}\right)\right\rangle=0
$$

and therefore that $G_{11}(\xi)$, which is clearly positive for small enough $\xi$, must become negative at large $\xi$. Thus there must be some intermediate value, $\xi_{0}$, for which $G_{11}\left(\xi_{0}\right)=0$. As shown in [11, 12] the finite size scaling behaviour of $\xi_{0}$ with $L=\sqrt{N}$ is related to the Hausdorf dimension of the surface:

1. $\xi_{0} \rightarrow$ constant as $L \rightarrow \infty$; this is the crumpled phase and has $d_{H}=\infty$. If there is only one mass scale then $\xi_{0} \propto m^{-1}$ where $m$ is the mass gap.

2. $\xi_{0} \rightarrow L^{1-\eta}, 0 \leq \eta \leq 1$, as $L \rightarrow \infty$; then $d_{H}=4(2-\eta)^{-1} \leq 4$ and the system is not crumpled.

In this work we are examining the crumpled phase so 1) is the relevant scenario. When $L$ is finite, $\xi_{0}$ will saturate as $\kappa$ is increased towards the critical point because it cannot in any case exceed $L / 4$ and so we expect to see finite size effects. The values of $\xi_{0}$ extracted from $G_{11}$ on $16^{2}, 32^{2}$ and $64^{2}$ lattices are plotted in fig.4 and show these effects clearly. In order to extract the true value of $m$ near the critical point it is necessary to have a model for the finite size effects. Previous work 12, 13] has shown that the data for $G_{11}$ at long distances in the crumpled phase is quite well accounted for by supposing that it is described by the effective Lagrangian

$$
\mathcal{L}_{\text {free }}=\frac{1}{A(\kappa)}\left(-\frac{1}{2} m(\kappa)^{2} \mathbf{X} \cdot \nabla^{2} \mathbf{X}+\mathbf{X} \cdot\left(\nabla^{2}\right)^{2} \mathbf{X}\right)
$$

which leads to the momentum space correlation function

$$
G_{i j}(\vec{p})=\left\langle\mathbf{t}_{i}(\vec{p}) \cdot \mathbf{t}_{j}(-\vec{p})\right\rangle=A(\kappa) \frac{p_{i} p_{j}}{p^{2}\left(p^{2}+m(\kappa)^{2}\right)} .
$$

Using this asumption we can remove the finite size effects by fitting (23) to the data in the region where $G_{11}(\xi)<0$. But, how good is the assumption?

In fig.5 we show a sequence of measured $G_{11}$ at different $\kappa$ values on a $64^{2}$ lattice together with the fit described above. As can be seen, at large distances the data is well fitted by (23) right up to the phase transition. The last picture at $\kappa=0.82$ shows completely different behaviour; the surface is no longer in the crumpled phase. This phenomenon provides us with an upper bound on $\kappa_{c}$ which can be used to constrain the fits for critical exponents. No error bars are shown on the data. The errors are smaller than the symbols on the figure but the correlation function at successive $\xi$ values is highly correlated; the fits of (22) were made by minimizing the $\chi^{2}$ function obtained using a correlated error analysis and SVD. However we do not actually know the true probability distribution of this data and so the errors on the values of $A$ and $m$ were obtained using a bootstrap procedure; all the values so obtained are listed in Table 1 together with the actual number of degrees of freedom (as determined by the SVD) contributing to the fits. In fact the 
Table 1: $\mathcal{L}_{\text {eff }}$ parameters deduced from correlation functions.

\begin{tabular}{|c|c|c|c|c|c|}
\hline$\kappa$ & $m$ & $A$ & d.o.f. & $\tilde{m}$ & $g$ \\
\hline \hline 0.7400 & $.348(2)$ & $8.95(7)$ & 15 & - & - \\
0.7500 & $.313(2)$ & $8.64(10)$ & 16 & - & - \\
0.7600 & $.273(2)$ & $8.02(8)$ & 17 & - & 5.8 \\
0.7700 & $.241(2)$ & $7.66(5)$ & 18 & - & 4.7 \\
0.7750 & $.220(2)$ & $7.42(6)$ & 17 & - & - \\
0.7775 & $.203(2)$ & $7.21(8)$ & 18 & $.204(4)$ & - \\
0.7800 & $.201(2)$ & $7.28(6)$ & 18 & $.203(3)$ & 3.7 \\
0.7825 & $.192(3)$ & $7.10(8)$ & 19 & $.192(3)$ & - \\
0.7850 & $.174(2)$ & $6.84(5)$ & 19 & $.174(2)$ & - \\
0.7875 & $.167(2)$ & $6.76(6)$ & 19 & $.165(3)$ & - \\
0.7900 & $.155(3)$ & $6.59(7)$ & 19 & $.153(4)$ & 3.0 \\
0.7925 & $.144(2)$ & $6.47(6)$ & 19 & $.143(3)$ & - \\
0.7950 & $.130(2)$ & $6.29(3)$ & 19 & $.129(2)$ & - \\
0.8000 & $.110(3)$ & $6.06(8)$ & 19 & $.108(5)$ & 1.8 \\
0.8050 & $.078(5)$ & $5.70(10)$ & 18 & $.076(7)$ & - \\
0.8075 & $.057(4)$ & $5.47(7)$ & 19 & $.055(6)$ & - \\
0.8100 & $.039(7)$ & $5.36(8)$ & 18 & $.037(12)$ & 1.1 \\
0.8110 & $.030(4)$ & $5.33(6)$ & 19 & $.029(11)$ & - \\
0.8125 & $.011(11)$ & $5.27(3)$ & 19 & $.012(10)$ & - \\
\hline
\end{tabular}

data is of high quality and, as can be seen from Table 1, the number of contributing degrees of freedom is large so our procedure should be reliable. There is however one possible source of systematic error. At short enough distances the two point function is not well described by (23) and so it is necessary to decide the minimum $\xi$ at which to attempt the fit. Obviously there are different ways of doing this and it is not clear that they will give the same results. To check on this we tried some other procedures than fitting to the region where $G_{11}(\xi)<0$. The $\tilde{m}$ column in Table 1 shows the results of fitting in the range $\xi \geq 10$ for all $\kappa$ values. As can be seen the $\tilde{m}$ values are always very close to the $m$ values but the estimated statistical errors are somewhat larger (probably because the number of contributing degrees of freedom is generally smaller).

The measured values of $m$ for $64^{2}$ lattices are plotted in fig.6. We have much higher statistics than in 13 and, crucially, reliable measurements for $\kappa$ above 0.8 . The consequence of this is that the best fit of the data to

$$
m=a\left(\kappa_{c}-\kappa\right)^{\nu}
$$

quoted in [13] $\left(\kappa_{c}=0.821(5), \nu=0.89(7)\right)$ is now seen to be a poor fit to the data very close to the critical point. Analysis of the new data set gives

$$
\begin{aligned}
\nu & =0.71(5) \\
\kappa_{c} & =0.814(2)
\end{aligned}
$$


with a $\chi^{2}$ per degree of freedom of 1.75. This fit is shown by the middle of the three lines in the figure while the outer lines show the fits with $\nu$ taken such that the $\chi^{2}$ per degree of freedom is 2.75 . If the $\tilde{m}$ values are fitted instead then we find

$$
\begin{aligned}
\nu & =0.68(10) \\
\kappa_{c} & =0.813(3)
\end{aligned}
$$

with a $\chi^{2}$ per degree of freedom of 0.98 . The central values are in good agreement with (26) but the errors are larger reflecting the larger errors in the $\tilde{m}$ values.

It is encouraging that this new direct measurement of $\nu$ is apparently in much better agreement with the result obtained by finite-size scaling of the specific heat peak [13, 19] but salutary in that it is necessary to work very close to the critical point to obtain this agreement.

\section{The specific heat}

There is a potential ambiguity in our treatment of the specific heat depending upon whether we calculate it with the original action $S_{e c}$ (3) or the action $\bar{S}_{e c}(13)$ used in the actual simulation; we have analysed both with very similar results for critical exponents so here we will just present the results for $\bar{S}_{e c}$. The specific heat arising from the gaussian part of the action is analytic as a function of $\kappa$ so we subtract it and define the specific heat to be

$$
C=\left\langle S_{\text {sym }}^{2}\right\rangle-\left\langle S_{\text {sym }}\right\rangle^{2}-\beta^{4}\left(\left\langle S_{G}^{2}\right\rangle-\left\langle S_{G}\right\rangle^{2}\right)
$$

Our data is shown in fig.7. The heights and locations of the peaks for various system sizes can be analyzed using the standard finite size scaling relations [21]

$$
\begin{aligned}
C_{\max } & =a+b L^{\omega} \\
\kappa_{\max } & =\kappa_{c}+b^{\prime} L^{-\frac{1}{\nu}}
\end{aligned}
$$

where $a, b$ and $b^{\prime}$ are constants and it is expected that $\omega=\alpha / \nu$. Unfortunately our systems have two different shapes (this is forced upon us by our FFT implememtation which only allowed $N$ to be a power of 2) and the coefficients $b$ and $b^{\prime}$ might depend upon the shape; ignoring this for the moment and taking $L=\sqrt{N}$ we find

$$
\omega=0.74(20) .
$$

This can be compared with the value $\omega=0.76$ deduced from the symmetric lattice data alone so we conclude that at this level of accuracy it is justifiable to include all the data in order to obtain an error estimate. Although the central value for $\omega$ differs from [19] who found $\omega=1.11(11)$ it is clear that with present statistics the error on $\omega$ is rather large. The errors on the measured values of $\kappa_{\max }$ are sufficiently large for the smaller lattice sizes that (31) is not very effective for deducing the correlation length exponent; however the data is consistent with the value $\nu=0.71(5)$ derived from the correlation functions. 
Assuming the hyperscaling result $\alpha=2(1-\nu)$ and the finite size scaling result $\omega=\alpha / \nu$ we find that

$$
\begin{aligned}
& \nu=0.73(6) \\
& \alpha=0.54(10)
\end{aligned}
$$

Using the measured value of $\omega$ and our independent measurement of $\nu(26)$ we obtain

$$
\alpha=\omega \nu=0.53(14) \text {. }
$$

Alternatively $\alpha$ can be found from (26) by assuming hyperscaling in which case

$$
\alpha=2(1-\nu)=0.58(10)
$$

The agreement between these methods of determination is very satisfactory but the value of $\alpha$ is much larger than that estimated by a direct fit to the specific heat in 13. However the shape of the specific heat curve does not determine $\alpha$ very accurately and a fit of the form

$$
C=a+b\left(\kappa_{c}-\kappa\right)^{-\alpha}+c \kappa
$$

to the full $64^{2}$ data for $\kappa \leq 0.795$ with $\kappa_{c}=0.814$ (26) and $\alpha=0.58$ (36) yields a $\chi^{2}$ per degree of freedom of 1.7; the line through the $64^{2}$ data points in fig.7 shows this fit. Thus we can now conclude, on the basis of much greater statistics than in [13], that all the different methods of analyzing the critical behaviour are broadly consistent.

\section{Normal-Normal Correlation Functions}

The normal-normal correlation function defined by

$$
G_{\mathbf{n}}(\vec{\xi})=\langle\mathbf{n}(\vec{\xi}) \cdot \mathbf{n}(0)\rangle
$$

where

$$
\mathbf{n}(\vec{\xi})=\mathbf{t}_{1}(\vec{\xi}) \times \mathbf{t}_{2}(\vec{\xi})
$$

is interesting because it tells us something about the four-point couplings in the theory. If the model were described exactly by the free field Lagrangian (22) then this correlation function would be given by

$$
G_{\mathbf{n}}(\vec{\xi})=\frac{2}{3}\left(G_{11}(\vec{\xi}) G_{22}(\vec{\xi})-G_{12}(\vec{\xi}) G_{21}(\vec{\xi})\right)
$$

At large distances this gives

$$
G_{\mathbf{n}}(\vec{\xi}) \sim-\frac{1}{|\vec{\xi}|^{4}}
$$

Corrections to this behaviour can take two generic forms. Firstly the power of the scaling law at large $|\vec{\xi}|$ may change or the coefficient of the $|\vec{\xi}|^{-4}$ behaviour could be 
different from that predicted by (40); this is the behaviour that would be obtained if a relevant operator other than those in $\mathcal{L}_{\text {free }}$ were present. On the other hand an irrelevant operator would generate deviations from (40) which fall off faster than $|\vec{\xi}|^{-4}$ at large distances. For example, adding interaction terms so that the effective Lagrangian is

$$
\mathcal{L}_{\text {eff }}=\mathcal{L}_{\text {free }}+\frac{g(\kappa)}{A(\kappa)^{2}}\left|\partial_{1} \mathbf{X} \times \partial_{2} \mathbf{X}\right|^{2},
$$

where $g$ is a dimensionless constant, gives a super-renormalizable theory and at large distances the induced correction to $G_{\mathbf{n}}(\vec{\xi})$ falls off as $|\vec{\xi}|^{-6}$. In fact our data is fully consistent with the picture that in the crumpled phase there are only interaction terms of a super-renormalizable nature.

In fig. 8 we show the measured $G_{\mathbf{n}}(\vec{\xi})$ for $\vec{\xi}$ lying along the x-axis at $\kappa=0.77$ on a $64^{2}$ lattice; the "free" line shows the form predicted by $\mathcal{L}_{\text {free }}$ using the parameters $A, m$ determined by the fits to $G_{11}$ described in section 4 . Clearly at distances $|\vec{\xi}|>18$ the predicted form is consistent with the measured one while at shorter distances there is a large discrepancy. This discrepancy can largely be explained by assuming a four-point coupling of the form (42) and computing the diagram shown in fig.9. The "interacting" line in fig.8 shows the computed $G_{\mathbf{n}}(\vec{\xi})$ obtained in this way with $g$ tuned to 4.7 which gives the best fit. As can be seen this fits the data much better at short distances but makes little difference to the long distance tail. As we move closer to the critical point it is necessary to look at larger distances to see the asymptotic behaviour. The behaviour of $G_{\mathbf{n}}(\vec{\xi})$ at $\kappa=0.79$ is shown in fig.10. On a $64^{2}$ lattice there is now a discrepancy between the data and the free field prediction out to distances $|\vec{\xi}| \simeq 25$. the picture also shows what happens on a $128^{2}$ lattice. Unfortunately the data is not very good but there is no evidence for a systematic deviation from (40) at large distances. The two curves showing the predictions of (42) on $64^{2}$ and $128^{2}$ lattices also show that there are significant finite size effects on the $64^{2}$ lattice at this $\kappa$; clearly using the $128^{2}$ data to estimate $g$ would yield a smaller value.

The values of $g$ deduced at some $\kappa$ values are shown in Table 1 . These values should be treated with some care because it is clear that there is a systematic discrepancy between the data and the prediction even when the interaction term is included; this makes it difficult to estimate an error for the values of $g$ quoted but it should be taken to be at least of order $20 \%$. It can be seen from the table that the value of $g$ is apparently falling as $\kappa$ approaches $\kappa_{c}$ so there is no indication for the appearance of a relevant perturbation away from $\mathcal{L}_{\text {free }}$ as the system approaches the critical point.

Finally we note that because the corrections to $\mathcal{L}_{\text {free }}$ are apparently superrenormalizable their presence does not affect our analysis of the two point function. At $O(g)$ only the $m$ parameter is renormalized and the form of the two point function is unaffected; $O\left(g^{2}\right)$ contributions will affect $G_{i j}$ at short distances but it is clear that there is a substantial range of $\xi$ for which they can safely be ignored. 


\section{Discussion}

We have determined the critical exponents $\alpha$ and $\nu$ by several different methods involving measurements of correlation functions and of the specific heat and making various theoretical assumptions. All the results obtained are in good agreement with each other. However in order to obtain this agreement it is necessary to make measurements with small statistical errors very close to the critical point and the nature of the analysis is slightly delicate. To obtain manifestly assumption-free measurements of the exponents would clearly require the use of much bigger lattices and need very much larger amounts of computer time than we have used.

For the first time, and as a direct consequence of our high statistics, we have been able to make a convincing measurement of the normal-normal correlation function. The consistency of our results with the idea that the effective field theory at the critical point is $\mathcal{L}_{\text {free }}$ means that there is still an unresolved puzzle concerning these models. At the critical point the free energy density $f$ on a strip of infinite length and finite width $L$ with periodic boundary conditions is expected to behave as

$$
f=f_{\infty}+\frac{\pi A}{6 L^{\rho+1}}+\ldots
$$

If the critical point is conformally invariant then $\rho=1$ and $A$ is the central charge [23]. Attempts have been made to measure this effect [22, 19]; assuming $\rho=1$ these workers all found that $A<1$. On the other hand $\mathcal{L}_{\text {free }}$ (which is not conformally invariant) predicts that $\rho=1$ and $A=6$. Whether or not the critical point is conformally invariant these two results are apparently inconsistent. However these measurements of $c$ are all done on strips that are rather narrow, the widest strip being only twenty lattice spacings wide. From our measurements of $G_{n}$ at $\kappa=0.79$ we know that physics differs substantially from the asymptotic behaviour implied by $\mathcal{L}_{\text {free }}$ (fig.10). Thus, if one believes the scenario implied by $\mathcal{L}_{\text {eff }}$ then at $\kappa$ values less than 0.02 away from the critical point one would not expect to see the correct asymptotic behaviour of (43). In fact these measurements are done as close to the critical point as possible so it may be that they are misleading. Of course, it is equally possible that the $\mathcal{L}_{\text {eff }}$ scenario is incorrect; our measurements are consistent with it but they are not accurate enough at very large distances to rule

out other possibilities. To make further progress it will probably be necessary to make renormalization group studies of this model.

I am grateful to Domenec Espriu for his comments on this work. These simulations were done on a Meiko i860 Computing Surface and some of the i860 assembler routines were supplied by C.Michael. Stephen Booth of the Edinburgh Parallel Computing Centre, Mike Brown of the Edinburgh University Computing Service and Peter Stephenson ensured the smooth running of this project. This research was supported by the UK Science and Engineering Research Council under grants GR/G 32779, GR/G 37132, GR/H 49191, GR/H 01243, GR/H 00772, GR/J 21200 and GR/J 21354 by the University of Edinburgh and by Meiko Limited. 


\section{References}

[1] W.Helfrich, Z. Naturforsch. 28C (1973) 693.

W.Helfrich, J. Phys.,46 (1985) 1263;

L.Peliti and S.Leibler, Phys. Rev. Lett. 54 (1985) 1690;

D.Foerster, Phys. Lett. A 114 (1986) 115;

R.K.P.Zia, Nucl. Phys. B 251, (1985) 676.

[2] V.G.Knizhnik, A.M.Polyakov and A.B.Zamolodchikov, Mod. Phys. Lett. A3 (1988) 819.

[3] F.David, Mod. Phys. Lett. A3 (1988) 1651;

J.Distler and H.Kawai, Nucl. Phys. B 321 (1989) 509.

[4] J.Ambjørn, B.Durhuus and J.Fröhlich, Nucl. Phys. B 257 [FS14](1985) 433.

[5] F. David, Nucl. Phys. B 257 [FS14](1985) 543.

[6] V.A. Kazakov, I.K. Kostov and A.A. Migdal, Phys. Lett. 157B (1985) 295.

[7] V.A. Kazakov, Phys. Lett. 119A (1986) 140; D. Boulatov and V.A. Kazakov, Phys. Lett. B 184 (1987) 247.

[8] J.Ambjørn, B.Durhuus, J.Fröhlich and T.Jonsson, Nucl. Phys. B 290 [FS20](1987) 480.

[9] H.Kleinert, Phys. Lett. B 174 (1986) 335;

A.M.Polyakov, Nucl. Phys. B 268 (1986) 406.

[10] Y.Kantor and D.Nelson, Phys. Rev. Lett. 58 (1987) 2774;

Phys. Rev. A 36 (1987) 4020.

[11] J.Ambjørn, B.Durhuus and T.Jonsson, Nucl. Phys. B 316 (1989) 526.

[12] R.G.Harnish and J.F.Wheater, Nucl. Phys. B 350 (1991) 861.

[13] J.F. Wheater and P.W. Stephenson, Phys. Lett. B 302 (1993) 447.

[14] M.Baig, D.Espriu, A.Travesset, Nucl. Phys. B 426 [FS] (1994) 575-600.

[15] J.F.Wheater, J. Phys. A 27 (1994) 3323-3353.

[16] F. David, Simplicial Quantum Gravity and Random Lattices, Saclay Physique Theorique preprint 93/028.

[17] J. Ambjørn, Fluctuating Geometries in Statistical Mechanics and Field Theory, preprint hep-th/9411179;

Recent progress in the theory of random surfaces and simplicial quantum gravity, talk given at Lattice94, preprint NBI-HE-94-55, hep-lat/9412006.

[18] M. Baig, D. Espriu and J.F. Wheater, Nucl. Phys. B 314 (1989) 609. 
[19] B. Jegerlehner and B. Petersson, Nucl. Phys. B (Proc. Suppl.) 34 (1994) 723.

[20] G.G.Batrouni et al, Phys. Rev. D 32 (1985) 2736;

A.Ukawa and M.Fukugita, Phys. Rev. Lett. 55 (1985) 1854.

[21] E.Domany, K.K.Mon, G.V.Chester and M.E.Fisher, Phys. Rev. B 12 (1975) 5025 .

[22] R.L. Renken and J.B. Kogut, Nucl. Phys. B 348, (1991) 580.

[23] J.L.Cardy, in Fields, Strings and Critical Phenomena eds. E.Brézin and J.ZinnJustin, North Holland, Amsterdam 1990.

\section{Figure Captions}

1. The value of $\sigma$ (13) required at different $\kappa$ values to yield $\rho=1$ (15) as determined on a $16^{2}$ lattice.

2. The ratio $\rho$ (15) determined on $32^{2}$ and $64^{2}$ lattices.

3. The bin size, $T_{b i n}$, estimated from the specific heat (17) as a function of $\kappa$.

4. The location of the zeroes of the correlation function $G_{11}$ as a function of $\kappa$ for different lattice sizes. Errors are smaller than the size of the symbols.

5. The correlation function $G_{11}$ in the region where it is negative for different $\kappa$ values on a $64^{2}$ lattice. The dashed lines show a fit of the form of (23) with the parameters given in Table 1. Errors are discussed in the text.

6. The mass gap $m$ (see Table 1) plotted as a function of $\kappa$. The three lines are discussed in the text.

7. The specific heat as a function of $\kappa$ for different lattice sizes. The line is the fit of (37) to the $64^{2}$ results with the constraint that $\kappa_{c}=0.814$.

8. The correlation function $G_{\mathbf{n}}$ at $\kappa=0.77$ on a $64^{2}$ lattice. The "free" line is that predicted by (40) while the "interacting" line is that predicted by (42) with the parameters given in Table 1.

9. Correction to $G_{\mathbf{n}}$ at $O(g)$. The open circles represent $\mathbf{n}$ sources, the filled circle a vertex of the form (42) and the lines $G_{i j}$ s.

10. The correlation function $G_{\mathbf{n}}$ at $\kappa=0.79$ on a $64^{2}$ and $128^{2}$ lattices. The "free" line is that predicted by (40) while the "interacting" lines are those predicted by (42) with the parameters given in Table 1 . 
Figure 1:

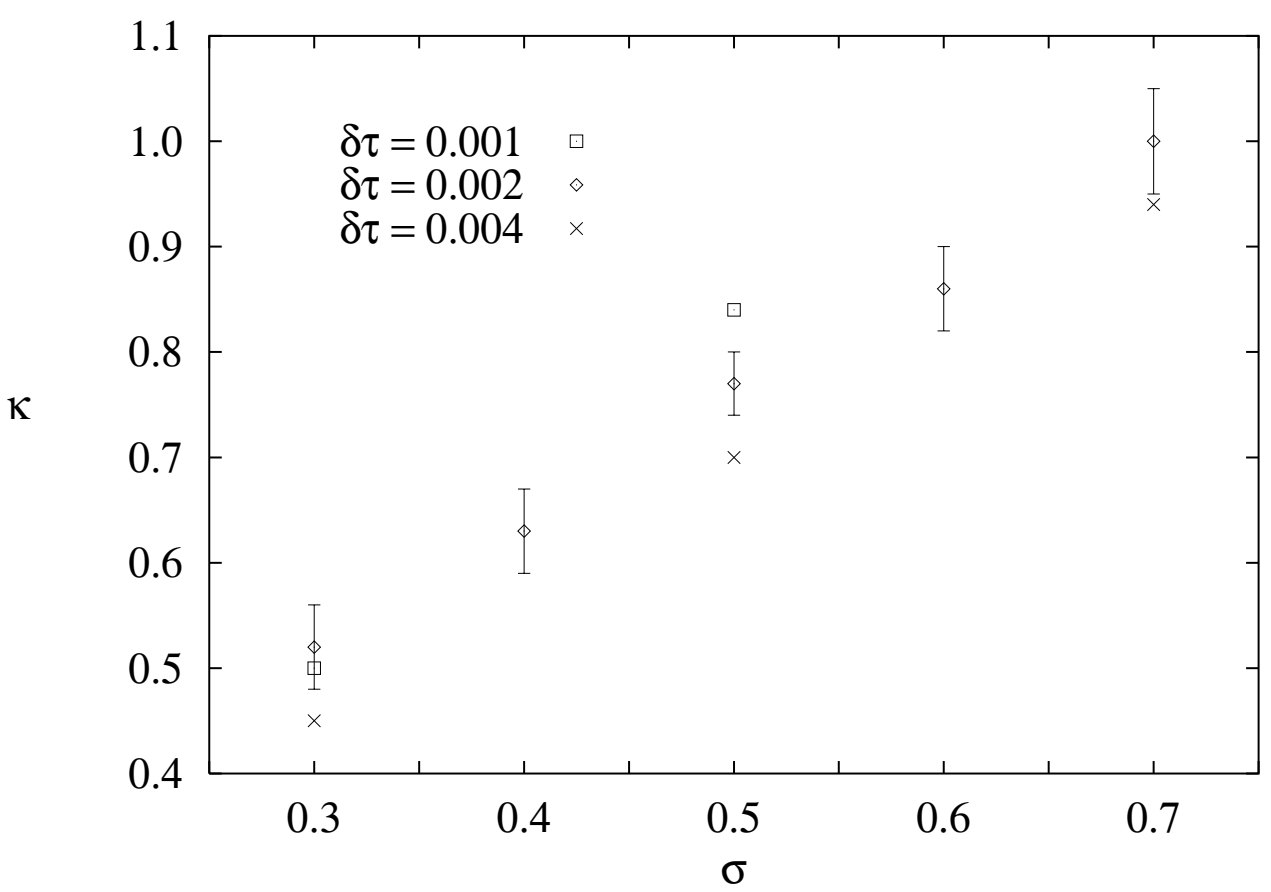

Figure 2:

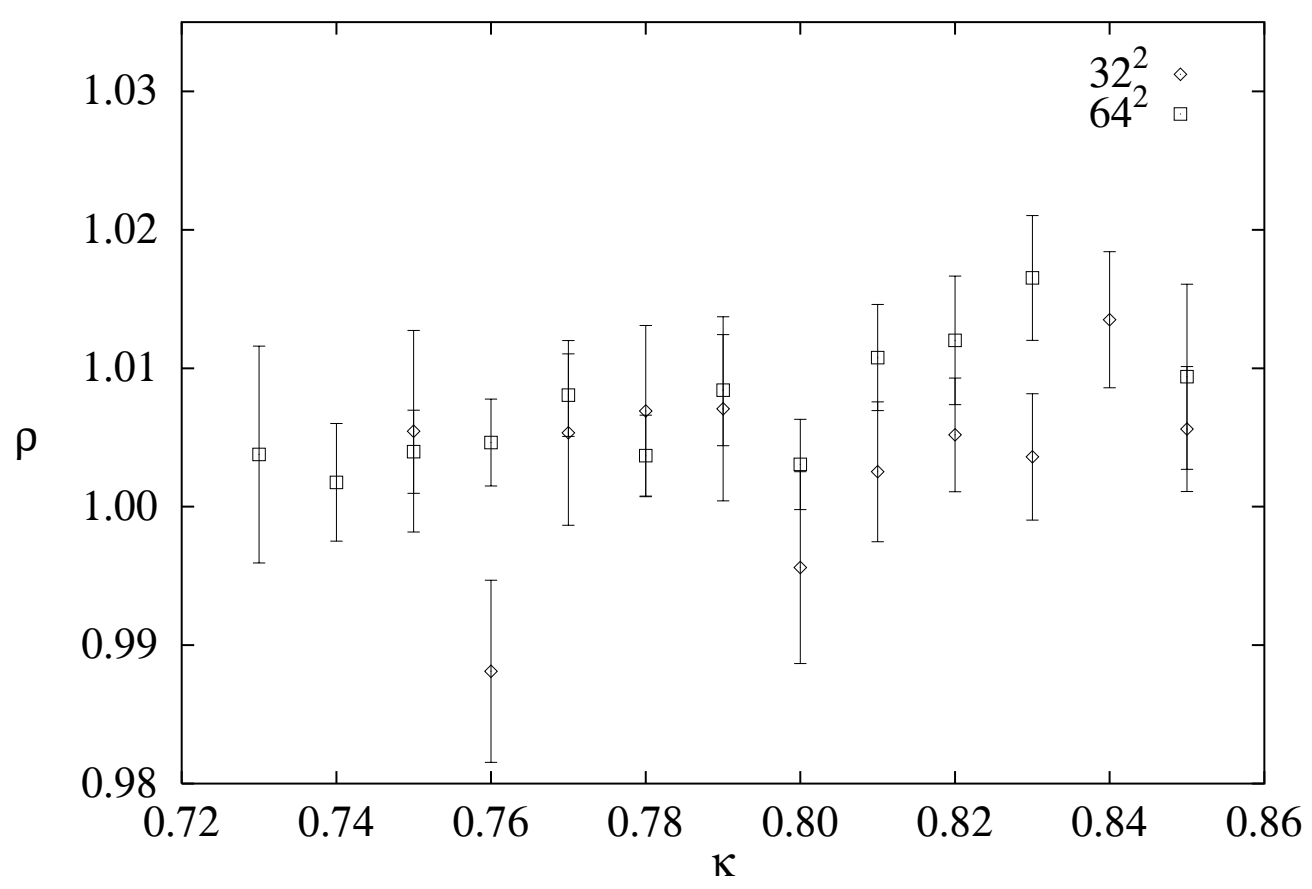


Figure 3:

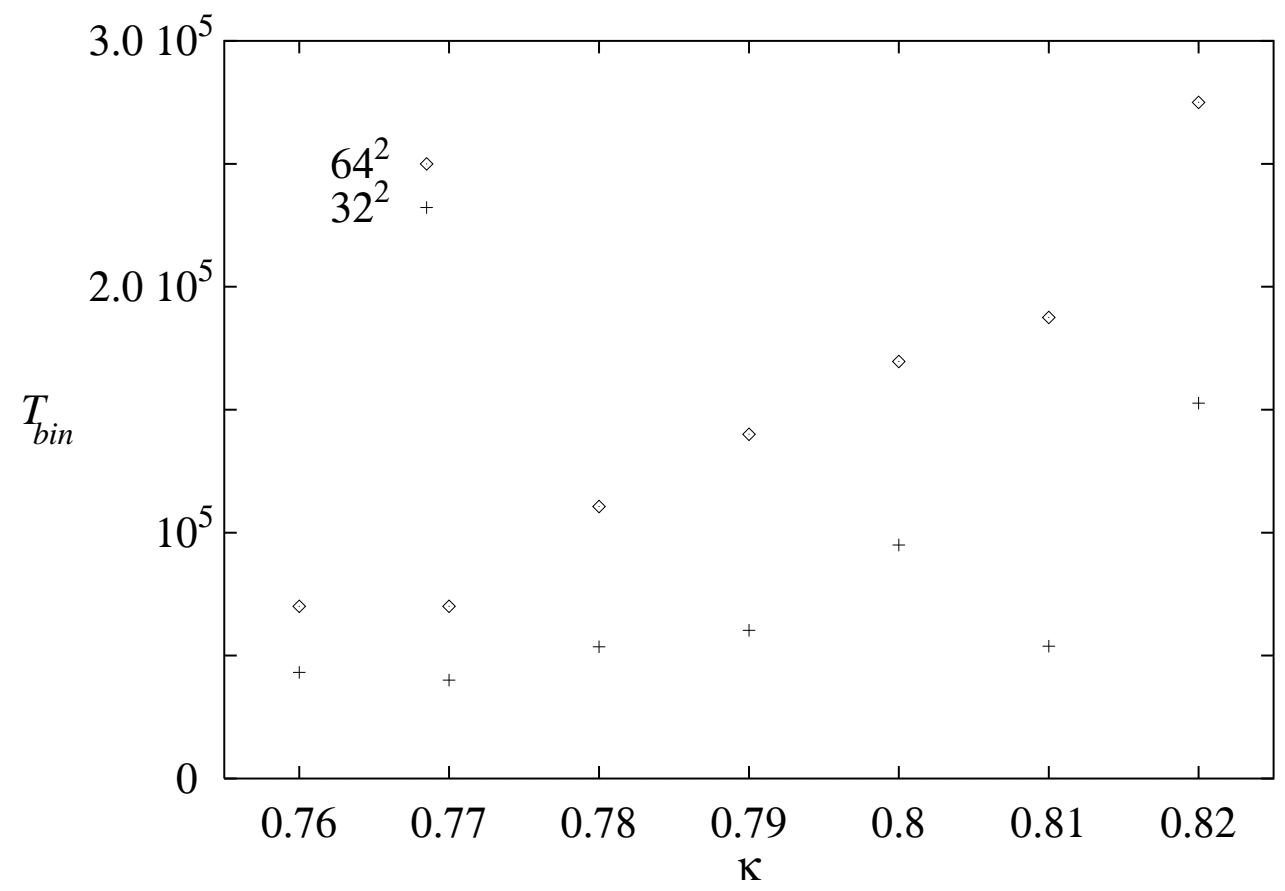

Figure 4:

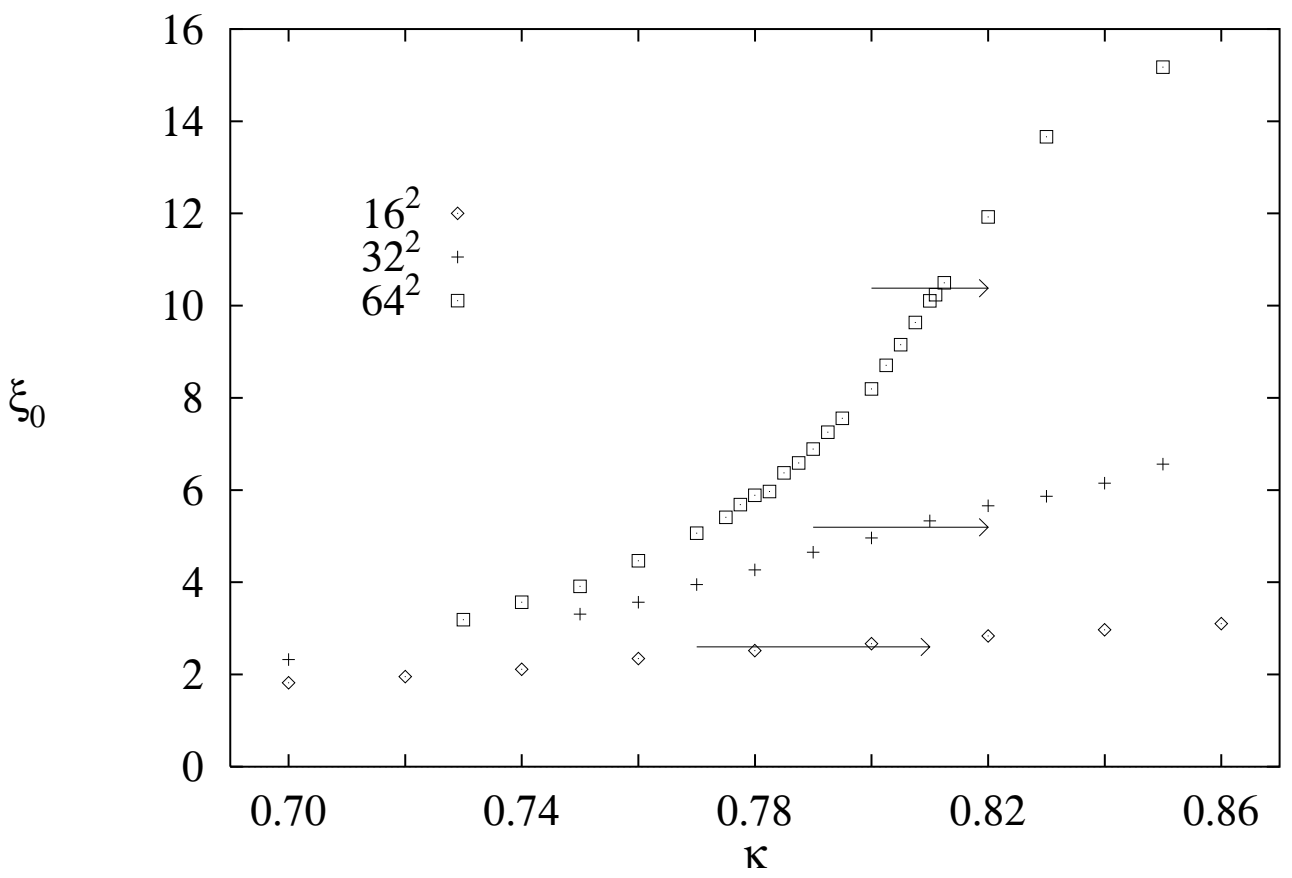


Figure 5:

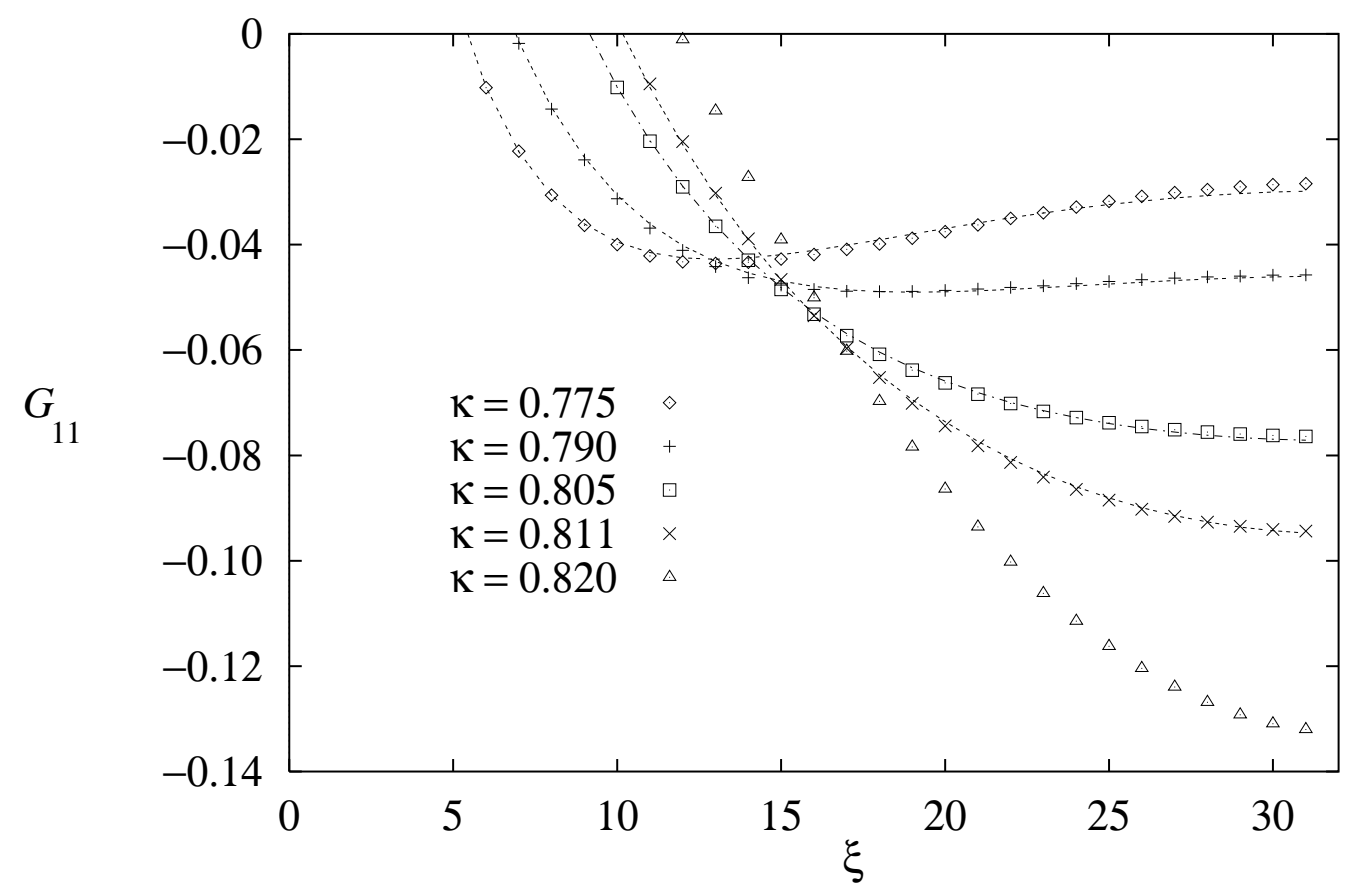

Figure 6:

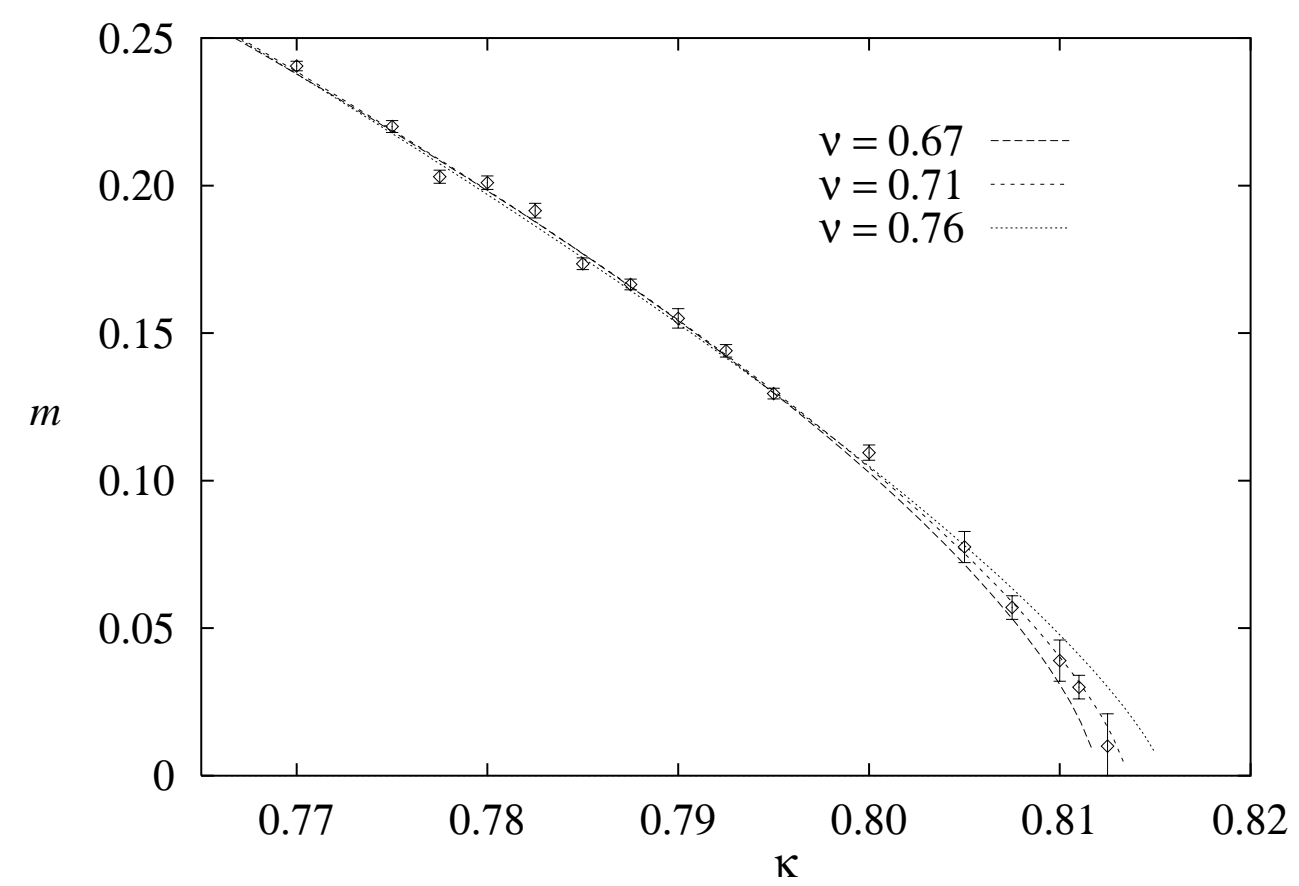


Figure 7:

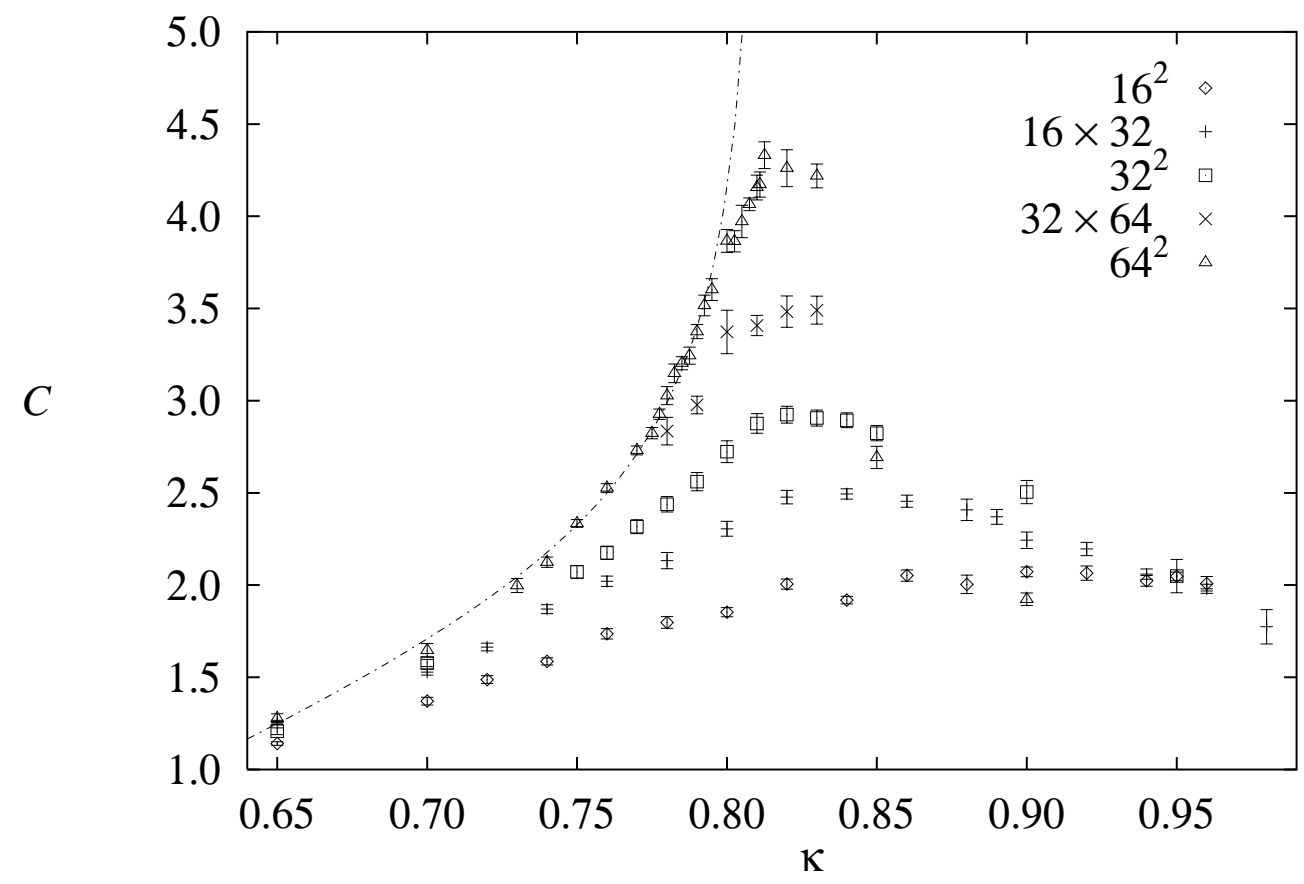

Figure 8:

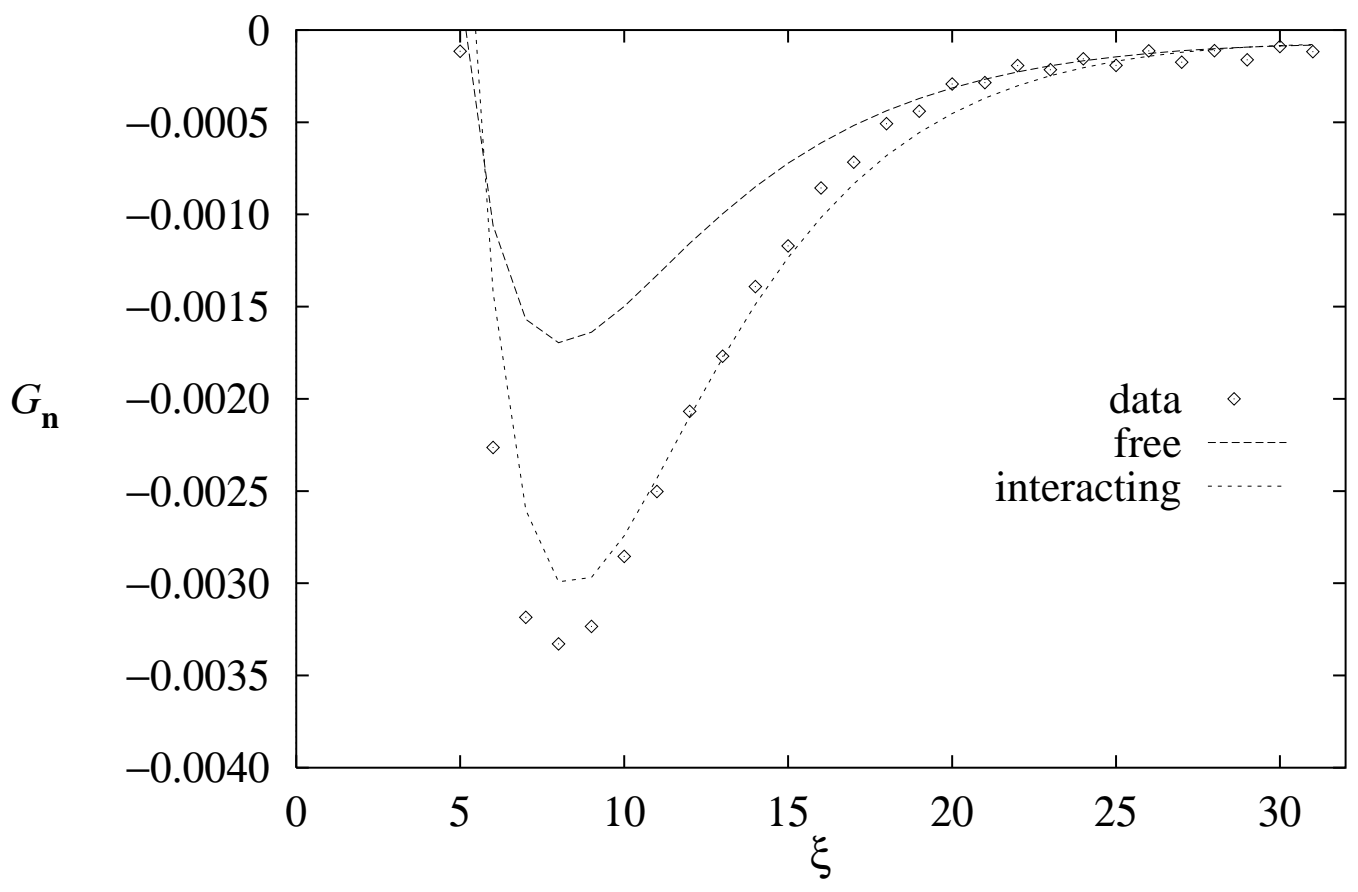


Figure 9:

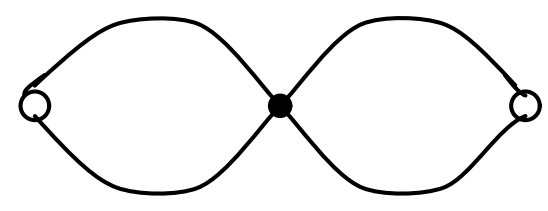

Figure 10:

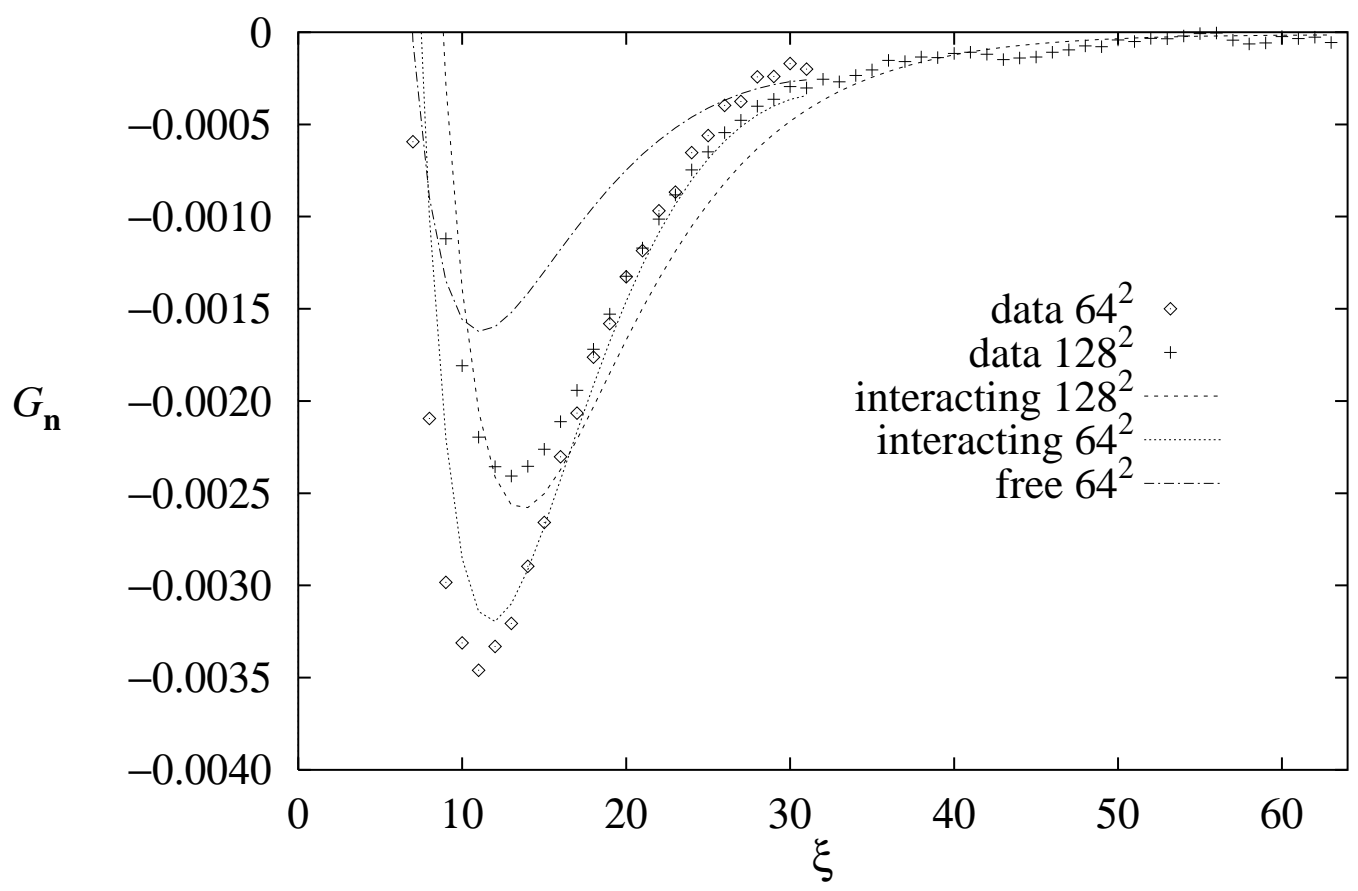

